\title{
The Social Sharing of Emotion as an Interface Between Individual and Collective Processes in the Construction of Emotional Climates
}

\section{Bernard Rimé* \\ University of Louvain}

The study of private emotional experiences reveals that an emotion is typically followed by social sharing. Additionally, the target's responses stimulate the sharing person's emotional expression. The reciprocal stimulation of interactants favors empathy and emotional communion. Consequences are two-fold: (1) the sharing process reactivates emotional arousal in both agent and target, and (2) it strengthens social bonds. Sharing targets also share what they heard with others, who then experience a similar need. Social sharing also develops when an emotional event strikes collectively. In this case, however, the propagation consequences are geared up in a spectacular manner both because there are as many sharing sources as there are members in the community and because every sharing reactivates felt emotions among interactants, thus reloading the propagation flow. It is argued that such chain reactions contribute to construct an emotional climate in the concerned community. It is predicted that emotion sharing would impact (1) on emotional climate in general; (2) on group cohesion and solidarity, with positive consequences for emotional climate; and (3) on collective memory, with potential consequences for emotional climate in the long run. Each of these predictions is detailed and illustrated with available empirical evidence.

Emotional climates reflect how individuals think the majority of others are feeling in a society's current situation (de Rivera, 1992; de Rivera \& Paez, this issue ). Emotional climates are objective in the sense that they exist apart from an Q1

* Correspondence concerning this article should be addressed to Bernard Rimé, University of Louvain, Place du Cardinal Mercier, 10, 1348 Louvain-la-Neuve, Belgium [e-mail: bernard.rime@ psp.ucl.ac.be]

I want to thank Dario Paez for his innumerable suggestions throughout the preparation of this article, which he would have coauthored if there had not been limitations in the number of contributions per author in this special issue.

307

C 2007 The Society for the Psychological Study of Social Issues 
individual's personal feelings. They often result from objective facts that create shared experiences. Emotional events that affect collectively a group or a community constitute a major source of influence for emotional climates. They exert this influence through the social processes they elicit and through the direct interactions that then develop among group members. Specifying how this works and thus describing potential interfaces between individual and collective process in the construction of emotional climates represents an important scientific challenge. This article intends to focus upon the role of the social sharing of emotion in this regard. This previously unsuspected microsocial process was first evidenced in the study of private emotional experiences (Rimé, Mesquita, Philippot, \& Boca, 1991). We will argue that when collective emotional events happen, the same process literally spreads across individuals and has a major impact on the construction of an emotional climate. We will first briefly review data showing that a person who has experienced an emotion is systematically inclined to communicate the event and to share related thoughts and feelings with others. We will then show that this process leads to considerable social and emotional consequences for members of the sharing person's social surroundings. A number of empirical predictions for emotional climates will follow from this review and the second part of the article will examine existing preliminary evidence in support of these predictions.

\section{Every Emotional Experience Elicits the Social Sharing of Emotion}

Private emotional experiences are generally followed by the social sharing of emotion, or evocation of the episode in a shared language to some addressee by the person who experienced it (Rimé et al., 1991). Thus, the affected person will talk with others about the event's emotional circumstances and his/her experienced feelings. This takes place during the hours, days, or even weeks and months following the emotional episode. It is modally a repetitive process and involves several recipients. More intense emotions are shared more recurrently, with more addressees, and the urge to share them extends on a longer period. This was first demonstrated in studies that relied upon autobiographic data. Participants had to recall an emotional experience from their recent past corresponding to a specified basic emotion (e.g., joy, anger, fear, shame, or sadness) and to answer questions about their sharing of this episode. In a review of eight studies of this type (Rimé, Philippot, Boca, \& Mesquita, 1992) involving in total 1,384 emotional episodes reported by 913 respondents ranging in age from 12 to 72 years, 88-96\% emotional episodes were socially shared. These proportions were independent of age and gender. Neither the type of basic emotion involved nor the valence of the emotional experience affected the proportion of shared episodes. Yet, emotional episodes involving shame and guilt were shared at a somewhat lesser degree (Finkenauer \& Rimé, 1998). The modal pattern for an emotion sharing was to be initiated early. Participants first shared the event on the day it happened in about 
$60 \%$ of the cases. Altogether, these data supported the view that "every emotion tends to be socially shared."

As autobiographic data are at risk of being unduly inflated and are open to reconstructive biases, the social sharing of emotion was examined further using other research methods. In follow-up procedures, participants were contacted at the time of their exposure to a specific emotional event and were then followed up for several weeks. Rimé, Finkenauer, Luminet, Zech, and Philippot (1998) reviewed six such studies involving respectively child delivery by young mothers, the loss of a close person, academic examinations, blood donation for the first time, and, among medical students, performing a first dissection of a human corpse. In the first week after the episode, sharing was manifested in each sample in about $90 \%$ of the cases, a rate which closely matched autobiographic studies. Marked decreases were then generally observed in the following weeks or months. Steeper extinction slopes occurred for less intense emotional events. Altogether these procedures precluding selective memory biases confirmed that people exposed to emotion engage in social sharing. Experimental induction of emotion by movie excerpts confirmed the causal link relating emotional exposure and the social sharing of emotion (Luminet, Bouts, Delie, Manstead, \& Rimé, 2000).

To sum up, available empirical studies abundantly document the fact that when people experience an emotion, they then talk about it whatever their gender, their education, their culture, or the type of emotion involved. However, data also reveal that the way people share their emotions varies as broadly as possible. Thus, parameters of the social sharing of emotion such as latency (how long ago did people start talking after the emotional episode?), recurrence (how often did they talk about a same episode?), or target (to whom did they talk?) have been found to vary considerably as a function of respondents' gender, education, culture, or as a function of the type of emotion they referred to (e.g., Rimé et al., 1998; SinghManoux \& Finkenauer, 2001). In sum, as regards the links between emotion and Q2 social sharing, existing research supports something about as general as the view that "when people are hungry, they eat." How they eat, what they eat, where they eat, with whom they eat with, and so forth is expected to vary considerably with situations.

\section{Social Consequences of Sharing an Emotion}

\section{Bonding and Emotional Fusion}

Observing targets of social sharing of emotion revealed that a typical interpersonal dynamic develops in such situations (Christophe \& Rimé, 1997). First, targets evidenced a marked interest and a high availability for shared emotional episodes. Second, confirming that hearing an emotional story is emotion-eliciting, the listeners' emotion varied in intensity as a function of intensity of the shared 
episode. Third, the behaviors they displayed also varied with intensity of the shared episode. For low intensity episodes, listeners' responses mostly consisted of verbal manifestations. With increasing intensity of the shared episode, a linear decrease of these verbal responses occurred together with a linear increase in nonverbal behaviors such as touching, body contact, taking into the arms, or kissing.

The typical interpersonal dynamic of emotion sharing can be sketched as follows. Person A, who experienced an emotion, shares it with B. The latter manifests a strong interest, which stimulates the sharing process. Person A consequently expresses emotions more and more, thus arousing emotions in B. The reciprocal stimulation leads to an enhanced empathy in Person B and, in the end, to an emotional communion between $\mathrm{A}$ and $\mathrm{B}$. The latter manifests a willingness to help and support Person A. If the shared episode is intense, B adopts a nonverbal communication mode, with body contact or touching. In this manner, B's liking for A increases. Similarly A, who is the focus of B's attention, interest, empathy, and support, feels enhanced liking for the latter. In sum, sharing emotion has the potential to bring interacting persons closer to one another. As sharing addresses predominantly intimates (Rimé et al., 1998), it is instrumental in maintaining, refreshing, and strengthening important social bonds.

There are notable exceptions to the general trend according to which social sharing interactions bring interactants closer to one another. Long ago, Pennebaker and Harber (1993) had observed that after an initial period of intensive sharing, Q3 victims of an earthquake progressively wore T-shirts that read, "Thank you for not sharing with me your earthquake experience." Studies on social interactions between patients suffering from a severe illness and healthy members of their social network (e.g., Herbette \& Rimé, 2004) documented situations that totally diverged from the sharing-intimacy model. Nevertheless, despite such important exceptions, both empirical and theoretical arguments support the generality of this model. Studies of self-disclosure interactions led to views exactly in the same direction (e.g., Reis \& Patrick, 1996). Thus, Laurenceau, Barrett, and Pietromonaco (1998, p. 1238) concluded that: "self-disclosure of emotion emerged as a more important predictor of intimacy than did self-disclosure of facts and information." A meta-analytic review of 94 studies about self-disclosure and liking led Collins and Miller (1994) to consider that (1) people who engage in intimate disclosures tend to be liked more than people who disclose at lower levels and (2) people like others as a result of having disclosed to them. The developmental background common to all human beings provides theoretical arguments in favor of the sharing-intimacy model. For every human being, the capacity to regulate one's emotion originated in the context of attachment relationships. Attachment indeed represents a resource that the infant activates when under stress (Bowlby, 1969). Thus, in stressful situations, attachment figures provided the child with presence, appeasement, contact, comfort, support, and meaning. It may then not come as a surprise that when later in life, individuals confront an emotional experience, their typical response is to 
turn to the social milieu to find among intimates ingredients such as appeasement, contact, comfort, support, and meaning.

\section{Propagation of Social Sharing}

As the social sharing of emotion arouses emotion in the target and as emotion elicits social sharing, it follows that those targets of social sharing should be inclined to share what they heard with third persons. In other words, a process of secondary social sharing is predicted. This was first documented by Christophe and Rimé (1997). In one of the studies, participants referred to a recent episode in which someone had shared with them an emotional episode resembling those in a list of 20. By random assignment, they received a list of low intensity episodes (i.e., lower end of a classic life events scale), a list of moderate intensity episodes (i.e., higher end of the same scale), or a list of high intensity episodes (i.e., a list of potential elicitors of post traumatic stress). They then rated their secondary social sharing of this episode. Consistent with the view that emotion elicits sharing, secondary social sharing was mentioned overall in $78.5 \%$ of the cases, with no significant differences across conditions. Consistent with what is observed for primary social sharing, frequency of secondary sharing was related to intensity, with more intense episodes eliciting more frequent repetitions of secondary sharing and with more partners.

These observations were recently replicated by Curci and Bellelli (2004). In one of the studies, volunteer students completed a daily diary for 15 days in which they had to report an episode shared with them by someone who had experienced it. The collected data showed that participants met such a situation on average every one day and a half (or in 10 out of the 15 days) so that 875 episodes were collected (302 positives and 573 negatives). The day they were heard, $54 \%$ of these episodes were secondarily shared, with no difference as a function of valence. This figure nearly matches those of primary sharing (i.e., around $60 \%$ on average, see Rimé et al., 1992). At the end of the diary procedure, Curci and Bellelli (2004) had their participants rate their total secondary sharing for each of the events they had reported. It was found that $55 \%$ of events that were not shared the day they were heard were shared on a later day. In this manner, $75 \%$ of all episodes collected in this study were shared. It can thus be assumed that some three-quarters of episodes personally confided to someone will then be shared by the latter with other people.

Social consequences of sharing an emotion do not end with secondary social sharing. A target of secondary sharing also experiences emotion when listening. This leads to an expectation of tertiary sharing from this person. Data indeed confirmed that episodes heard in a secondary sharing were shared again with one new listener for one third of participants and with several new listeners for another third (Rimé \& Christophe, 1997). In sum, emotional episodes open upon a process of spreading of emotional information through which they propagate very 
easily across social networks. It can be calculated that when a somewhat intense emotional event affects someone, 50-60 members of this person's community will be informed of it within the next few hours by virtue of this propagation. That such a process actually develops in real life was nicely confirmed in a field study wherein 33 college students visited a hospital morgue (Harber \& Cohen, 2005). Students' emotional reactions to this experience predicted how many people they told (primary sharing), how many people their friends told (secondary sharing), and how many people their friends' friends told (tertiary sharing). Within 10 days, nearly 900 people heard about this event through these cascading levels of social sharing.

Of course, as was demonstrated long ago in classic studies by Bartlett (1932), such a process of transmission of information is open to information transformations and reconstructions. Nevertheless, through the spreading of emotional information, members of a community are able to keep track of emotional experiences affecting their peers. In this manner, shared collective knowledge about emotional events and about emotional responses is continuously updated in function of new private experiences. As emotions occur when events are unexpected or unpredicted and require fast and appropriate responding, the spreading just described provides members of a group with a particularly effective prevention tool with regard to future emotion-eliciting situations.

\section{Social Sharing of Emotion and Emotional Climates}

Investigating the social sharing of emotion revealed two important characteristics of emotion. Both stand in sharp contrast with common assumptions. First, emotion is not a rapidly vanishing state that is limited to an emotional circumstance. Emotion impregnates the subsequent life of the affected person, sometimes for a very long time: the higher the intensity of the experience, the stronger its hold on later life. Second, emotion is not constrained within the boundaries of the affected individual. An emotional experience elicits its sharing, its sharing arouses emotion among social targets, and social targets propagate the sharing of the experience. In sum, emotion sharing reveals that emotions expand broadly both in time and in space. In other terms, emotions not only are anchored on social relations (de Rivera, 1992). Social consequences under the form of communication and interpersonal propagation are central to emotional episodes as well.

It should be added that these social effects of emotions still are considerably enhanced when the latter result from collective events, which spread through the media (e.g., collective loss victory, defeat, success, failure, catastrophe, accident, common threat...). When an emotional event strikes collectively a group, a community, or a society, indeed, spatial-temporal consequences are geared up in a spectacular manner. In private emotional experiences, sharing originates in one single source and reaches intimates of successive sharing agents in a wave that 
propagates essentially in an exocentric direction. In collective emotional events, there are as many sharing sources as there are members in the concerned community. Consequently, the propagation diffuses in as many directions. In private emotion, a progressive extinction of the propagation wave is expected as the social distance from the original source increases. In collective emotion, every sharing reactivates felt emotions among interactants and therefore reloads the propagation flow. In sum, a collective emotional event is likely to start chain reactions.

Such chain reactions can contribute to the construction of an emotional climate in the concerned community. Three processes of influences of social sharing on emotional climate, which are open to future empirical testing, can be formulated in this respect. They respectively regard the impact of emotion sharing (1) on emotional climate in general; (2) on group cohesion and solidarity, with positive consequences for emotional climate; and (3) on collective memory, with potential consequences for emotional climate in the long run. In the following sections, each of these three predictions will be specified and examined in the light of an illustrative example from the rare available data.

\section{Impact of Emotion Sharing on Emotional Climate}

As emotion sharing arouses emotional states both in the sharing person and the target, successive propagation waves elicited by a collective emotional event should progressively set up an emotional atmosphere in line with the emotional feelings elicited by an event. Indeed, emotional atmosphere was defined by de Rivera (1992) as the relatively short-term collective mood that occurs when group members focus on a common event. However, there are reasons to predict that when social sharing propagation waves about a given collective emotional event recur, such waves would also progressively affect the emotional climate or the way people perceive the current state of their group or nation. Sharing waves entail recurrent confrontation with the shared emotional episode and thus confer this event a particular saliency. In this manner, a recurrently shared collective episode gains a high mnemonic availability and becomes an easily accessible tool for appraising the present condition of the group or country. Thus, it is expected that successive social sharing propagation waves elicited by a positively valenced collective emotional episode would progressively set up a positive emotional climate. In a similar manner, a negative emotional climate would result from successive social sharing waves of a negatively valenced episode.

Let us first examine the prediction regarding the potential impact of the social sharing of positive emotional events upon emotional climate. No available data has actually tested the relation between the two variables. However, recent research opens clear perspectives in that direction. Sharing positive emotional episodes should activate or reactivate positive emotional feelings and memories among both the source and the target audience. Because of these positive emotional 
benefits, people should thus be motivated socially to share positive episodes further. In their diary studies, Curci and Bellelli (2004) indeed observed that people who had been exposed to the sharing of a positive emotion later initiated a secondary social sharing in a very abundant proportion. Research previously conducted by Langston (1994) shed a new light on the motives underlying the social propagation of positive emotional episodes. This author considered that the sharing of positively valenced episodes involves a "capitalization." Capitalization refers to the process of beneficially interpreting positive events. Thus, positive events are seen by people not as problems to be surmounted or coped with, but rather as opportunities upon which to seize or "capitalize." Capitalizing on a positive event can be achieved in at least three different ways: (1) by making it more memorable to the self (e.g., by marking the event's occurrence in some expressive fashion such as jumping, bragging, celebrating, etc.); (2) by seeking social contacts and letting others know about the event; and (3) by maximizing the event's significance (e.g., by increasing one's perceived control of the event). In two different studies, Langston (1994) found that communicating a positive event to others was indeed associated with an enhancement of positive affect which largely exceeded the benefits due to the valence of the positive events themselves. A capitalization effect was thus clearly evidenced. Gable, Reis, Impett, and Asher (2004) confirmed these findings. Additionally, they examined the role played by the way a social sharing audience reacts to the sharing of a positive emotion. They found that close relationships in which one's partner typically responds enthusiastically to a "capitalization" sharing were associated with higher relationship well-being (e.g., intimacy, daily marital satisfaction). Thus, sharing positive emotions not only increases positive affect at the intrapersonal level, but it also enhances social bonds. These results obtained from the study of interpersonal relationships open perspectives with regard to potential consequences of the sharing of a positive collective event for emotional climate. Collective emotional events such as victories, collective successes, collective good fortune, wins of sport teams, and deeds of group members lend themselves to capitalization processes by the community as a whole as well as by every individual member. Such positive collective events lend themselves particularly well to chain reactions in the propagation flow as described in the previous section. The process will first affect the temporary collective mood state of the concerned group or nation, thus modifying the momentary emotional atmosphere. However, lay observation reveals that the capitalization of positive collective events is everything but a one shot process. To illustrate, when in 1998 the French national soccer team won the World Cup, for years it became-for an entire nation-a favorite topic of social sharing supported by innumerable collective recalls in the media or in collective celebrations at later sport events. This collective episode thus gained and maintained for a very long time an exceptional mnemonic availability in the French population. Though no empirical data tested its impact upon emotional climate, there is little doubt that the memory of this 
collective triumph largely affected the way French people appraised the condition of their nation in the weeks, months, and even years that followed it.

As regard the prediction regarding the potential impact of the social sharing of negative collective emotional events upon emotional climate, some data in support of our prediction exist. They were provided by investigations of the social sharing of colonial wars in Africa among Portuguese students two decades after these wars had ended (Marques, Paez, \& Serra, 1997). The wars in which Portugal was involved against liberation movements of its three former African colonies lasted from 1961 to 1974 and engaged 280,000 troops in combat. They showed many similarities with the U.S. war in Vietnam, with civilian abuse, tough survival conditions, and atrocities proper to guerrilla warfare. Very high rates of post traumatic disorders resulted among Portuguese veterans. Yet, these troubles were never officially recognized by the Portuguese government as consecutive to wars, so that sufferers received neither economic compensation nor appropriate medical and social support. As in the U.S., there were many internal attempts against the continuation of this war. However, the Portuguese government was not democratic at that time and these attempts faced institutional repression from the state police.

Marques et al. (1997) reported two studies conducted in 1993 and 1994 in Portugal on youths who were not yet born at the time of the war. In the first one, participants' perception of veterans and of the Portuguese involvement in the colonial war was assessed. They reported on their rumination, re-evaluation, inhibition, and social sharing relative to this topic. They also rated their perception of their country's current emotional climate. In addition, they were asked whether during the last 40 years, themselves or a member of their family had been involved as victims or actors in violent acts such as torture or war. Overall, $21 \%$ of all respondents reported that close acquaintances were both victims and actors, $12 \%$ reported that these acquaintances were victims but not actors, and $18 \%$ reported that acquaintances were actors but not victims of political violence. Results revealed that the more respondents talked about a member of their family or an acquaintance having been either a victim of violence or an actor in this kind of violence, the more negatively they evaluated their country's emotional climate. As from 1975 onwards, Portugal was not directly involved in a war; as torture was eradicated, the young participants' responses necessarily regarded social memories held by them about a remote past. This revealed that the transgenerational sharing of traumatic past events reinforces a negative view of the contemporary society and manifested the impact of the past over the appraisal of the present.

In a second study, Marques et al. (1997) contacted 98 Portuguese students who all reported being acquainted with at least one veteran. Participants rated the frequency with which they heard someone talking about the war and how close such persons were to them. They also answered questions on (1) the way in which veterans around them shared their memories of the war, (2) the impact such sharing had on themselves and on the emotional climate of the veterans' 
families, (3) their own attitudes toward the war, and (4) how they accounted for the war. Results showed that the more participants heard about the war, the more they reported a negative mood ("unhappy," "worried," "sad," "anxious") in the veteran when he shared about the war. A similar phenomenon occurred with respect to the perception of the veteran's family emotional climate: the more they heard about the war, the more they perceived the family climate to be a negative one.

To conclude, consistent with our prediction, both studies on the sharing related to Portugal's colonial wars found that, two decades after the shared facts, the more participants were involved in such sharing, the more they rated negatively the present emotional climate either in their country (Study 1) or in their family (Study 2).

\section{Positive Consequence of Emotion Sharing for Emotional Climate}

In the previous section, we examined the impact that social sharing propagation waves can have on emotional climates via the process of emotional activation and reactivation that sharing emotion entails. We now move to another way through which social sharing waves can affect emotional climates. Sharing an emotion quite generally involves a social dynamic that is characteristic of collective rituals and that typically opens upon an emotional climate of mutual confidence and solidarity. We will first examine this social dynamic and its effects on climate in the framework of collective rituals. We will then show its counterpart in the framework of the social sharing of emotion. Finally, we will examine empirical observations in support of the impact of the described dynamic upon emotional climate.

Durkheim (1912) circumscribed the social dynamic that characterizes most collective rituals. He was also able to specify the consequences of this dynamic both for the concerned community and for each individual group member. Though originally focused on religious ceremonies, Durkheim's (1912) theory addressed a broad range of collective manifestations such as commemorations, celebrations, demonstrations, and the like. He stressed that such manifestations very generally favor a reciprocal stimulation of emotion among participants. The presence of group symbols (flags, emblems ... ), participants' collective expressions (singing, yelling, telling words or sentences, shared movements, music, and dance), and their momentary concentration on a common theme, are all factors of social rituals that lead individuals' consciousnesses to echo one another. Participants' salience of their individual self is lowered and their collective identity is enhanced. They experience unity and similarity. In sum, taking part in a ritual opens upon a state of emotional communion. Social rituals are thus particularly effective in enhancing participants' feelings of group belonging and of social integration. Beliefs and representations shared in the community are set at the foreground of their preoccupations. The high level of social consensus that prevails during the ritual contributes to the consolidation of participants' faith in their cultural beliefs and 
confidence in collective action. Consequently, when the ceremony ends, group members experience strong feelings of mutual confidence and solidarity. Social rituals thus have the potential to affect emotional climates deeply (for social rituals and emotional climate, see Paez et al. in this issue).

The social dynamic that develops in the course of naturally occurring situations of social sharing of emotion matches Durkheim's (1912) description of the socioemotional process involved in rituals. As was mentioned in our review of empirical findings about emotion sharing, the more a person expresses emotion when sharing, the more the sharing partner responds along socio-affective lines, thus providing the source person help and support, comfort and consolation, legitimization, attention, bonding, empathy, and so forth. Thus, through a very similar social dynamic resulting from reciprocal stimulation of emotion and from the empathy that ensues, sharing emotion has the same potential as collective rituals to bring participants closer to one another. We have seen earlier that this potential was very well documented empirically for social sharing and for emotional disclosure. We can safely conclude that, as is the case for collective rituals, sharing emotion contributes to maintaining, refreshing, and strengthening important social bonds. After a sharing round, participants' feelings of mutual confidence and solidarity are expected to be enhanced. The more intense the shared emotion was, the higher these social consequences should be.

In line with these considerations, we predict that the sharing wave provoked by a negative collective event would not only construct a negative emotional climate as was considered in prediction (1). As the interpersonal dynamic feeds up feelings of trust, confidence, and solidarity, the construction of a positive emotional climate is also expected. As the perceptions of a positive and of a negative emotional climate are relatively independent of one another, co-occurrence is possible. Such a cooccurrence was recently documented in a longitudinal study of Spanish citizens' psychological and social responses to the March 11 (M-11) terrorist bombing in Madrid in 2004 (Paez, Rimé, Martinez, \& Basabe, 2006).

Collective traumatic events such as those of September 11th in New York or of March 11th in Madrid elicited emotional arousal and social sharing of emotions in an overwhelming majority of inhabitants. Both in New York and in Madrid, a majority of respondents reported isolated symptoms of stress reaction and more than $80 \%$ of them spoke about the events and shared with others what they felt. In their longitudinal study, Paez et al. (2006) assessed the predictive validity of social sharing of emotion about M-11. One week after the M-11 bombing, then 3 weeks after it, and finally, 8 weeks after the events, a questionnaire was completed by 458 university students and their relatives in five Spanish regions. Assessed variables included social sharing of emotion, intensity of emotions, of rumination, of arousal, and of anxiety related to M-11, and current perception of the country's emotional climate. Results showed that 1 week after the events, participants had developed overabundant M-11-related social sharing. When they were reassessed 
3 weeks later and 2 months later, their emotional arousal and their social sharing manifested an important decrease as compared to initial values. At the 2-month follow-up, negative emotional climate and perceived solidarity also manifested a significant drop when compared to initial values. Further analyses revealed that, controlling for the initial level of intensity of emotional arousal, frequency of sharing about M-11 events and of hearing people sharing about them in the week after had a good deal of negative consequences. First, it contributed to sustain the events-related emotional arousal, as assessed by both intensity of emotions and anxiety/arousal scores, at least up until eight weeks after the events. Second, it contributed to sustain events-related mental rumination at least up until 3 weeks after the events. Third, it contributed to perpetuate the person's perception, in the weeks and months following the events, of a negative emotional climate in the national context. To sum up, consistent with our prediction (1), these results manifested that sharing emotion had fed people's emotional arousal and had ended up in constructing a negative emotional climate.

In spite of its apparent contradiction with prediction (1), prediction (2) was equally met in the results of the M-11 longitudinal study. Indeed, socially sharing emotions in the first week after M-11 events was found associated with a good number of indicators of social integration and of well-being assessed in later weeks. Thus, the initial sharing of emotions (1) reinforced the perception of social support at both the 3 and 8 weeks measurement, (2) reduced feelings of loneliness at both measurements, (3) enhanced positive affect at 3 weeks, (4) strengthened the perception of positive changes in reaction to trauma (posttraumatic growth), and (5) reinforced the perception of contentment, hope, solidarity, and confidence in the emotional climate at 8 weeks measurements. In addition, a structural equation analysis of these longitudinal data (see Paez et al., this issue), Q5 controlling for initial extent of participation in demonstrations for initial affect and for initial positive emotional climate, revealed that the initial level of talking about M 11 events was a significant direct and positive predictor of positive emotional climate assessed 2 months later. The latter result thus strongly supported our prediction (2).

Altogether, these results fit with theoretical views according to which the social sharing of emotions fulfils important functions with regard to affiliation and interpersonal empathy, to increased social cohesion, and to the reconstruction of positive social beliefs about the group (Rimé et al., 1998). They also fit with findings from experimental studies showing that even though sharing emotions was not conducive to emotional recovery, participants in sharing sessions reported a good number of positive benefits from such sessions (Zech \& Rimé, 2005). We can conclude that evidence already exists in support of the view that the social sharing of a negative collective emotion contributes to reinforce social integration, positive affect, social cohesion, and the construction of a climate of solidarity and hope. At the same time, by feeding emotional arousal among individuals who 
share, the social sharing of a negative collective event also contributes to construct a negative emotional climate.

\section{Consequences of the Sharing Process for Collective Memory}

Social sharing contributes to construct and consolidate shared knowledge about a collective experience and about people's emotional responses to this event. In doing so, it perpetuates the emotional climate associated with the sharing of the episode. To illustrate, one can expect a same recurrent emotional climate of fear, of anxiety, and of helplessness to be reactivated again and again in the American society every time the September 11 terrorist attacks would reappear on the media, whether for an anniversary or for any related circumstance. To our knowledge, no data is available to document the consequences that the construction of a collective knowledge about an event entails for a society's emotional climate. At least, however, the contribution of the sharing process to the construction of such collective knowledge is becoming documented more and more. An example is provided in a study that was conducted about an unexpected collective loss that occurred in Belgium (Finkenauer et al., 1998).

The Belgian king Baudouin died from a heart attack at the age of 62 in his vacation residence in Spain in July 1993. As the king was in good health in the preceding period, the news was unexpected and had an enormous impact on the Belgian population. Baudouin had been king for 42 years and was considered by many as a father of the nation. He had a strong unifying influence on a nation deeply divided by linguistic and cultural disagreements. Television and radio channels replaced their programs with broadcasts on the royal family and newspapers covered the event on about $60 \%$ of their pages. Some months later, Finkenauer et al. (1998) conducted a study on reactions to the king's death in a large group of French-speaking Belgians. Participants recalled the circumstances in which they first heard the news and answered questions on how they heard it, where they were when they heard it, and what their ongoing activity was at that moment. They also had to remember specific details about the event itself. In addition, they rated their emotional response to the event and their overt rehearsal of it (i.e., frequency of sharing and of following the media). A structural equation approach revealed that the intensity of participants' emotional response to this collective event did not directly determine their factual memory of the event. However, their emotional response had a particularly strong indirect influence on this memory and this influence was mediated by their extent of social sharing and social rehearsal The greater the level of emotionality, the more one had talked about the King's death and followed the media. In line with what was predicted here above, this social sharing of the collective event led to a better recall of the original event and consolidated people's memory. Similar findings occurred in the M-11 longitudinal study. The extent of social sharing developed 1 week after the bombing in Madrid 
predicted participants' correct recall and recognition of details of the collective traumatic event 2 months later (Paez et al., 2006).

\section{Conclusion}

We hope to have argued convincingly that theoretical perspectives linking people propensity to share their emotional experience and emotional climates as well as preliminary empirical observation opens promising perspectives for future investigation.

What we have accomplished in these pages are only first steps, and many issues raised in this article are in need of further development. To illustrate, in the course of a social sharing chain, the emotional experience itself may change as the account progresses from one person to another. Thus, an initial experience of sadness may lead a listener to feel anger and the next person to feel fear. Subgroups may develop in this manner around a same emotional event. At the fall of the Pinochet regime in Chile, opponents to this regime experienced joy and relief when sharing the event, whereas those who supported the dictatorship felt sadness, anger, and powerlessness. Also, blends of contrasting feelings may coexist in one and the same social group. After the 9/11 events in New York, the same people were experiencing fear and anxiety given the perspective the new attacks offered, pride in view of accomplishments of firemen and rescue services, compassion with regard to victims and their families, solidarity with peers, and at the same time, anger and feelings of revenge toward terrorists. It will be the task of future work to clarify the effects that such complex emotional situations can have for emotional climate.

Particular attention will have to be borne in this regard to the role played by political leaders and the mass media in influencing and orienting the emotions and feelings that spread across a population through social sharing after a collective emotional event. As was suggested by a concept such as a "war on terror" before the Iraqi war, or by the baneful broadcasts of "Radio 1000 collines" before the genocide in Rwanda, opinion leaders' rhetoric can heavily influence the climate generated by the social sharing.

\section{References}

Bartlett, F. C. (1932). Remembering: A study in experimental and social psychology. Cambridge, England: Cambridge University Press.

Bowlby, J. (1969). Attachment and loss, Vol. 1: Attachment. New York: Basic Books.

Christophe, V. \& Rimé, B. (1997). Exposure to the social sharing of emotion: Emotional impact, listener responses and the secondary social sharing. European Journal of Social Psychology, 27, 37-54.

Collins, N. L., \& Miller, L. C. (1994). Self-Disclosure and liking: A meta-analytic review. Psychological Bulletin, 116, 457-475.

Curci, A., \& Bellelli, G. (2004). Cognitive and social consequences of exposure to emotional narratives: Two studies on secondary social sharing of emotions. Cognition and Emotion, 18, 881-900. 
de Rivera, J. H. (1992). Emotional climate: Social structure and emotional dynamics. In K. T. Strongman (Ed.), International review of studies on emotion (pp. 197-218). New York: John Wiley \& Sons

Durkheim, E. (1912). Les formes élémentaires de la vie religieuse [The Elementary Forms of Religious Life]. Paris: Alcan.

Finkenauer, C., Luminet, O., Gisle, L., El-Ahmadi, A., Van der Linden, M., \& Philippot, P. (1998). Flashbulb memories and the underlying mechanism of their formation: Toward an emotionalintegrative model. Memory and Cognition, 26, 516-531.

Finkenauer, C., \& Rimé, B. (1998). Socially shared emotional experiences vs. emotional experiences kept secret: Differential characteristics and consequences. Journal of Social and Clinical Psychology, 17, 295-318.

Gable, S. L., Reis, H. T., Impett, E. A., \& Asher, E. R. (2004). What do you do when things go right? The intrapersonal and interpersonal benefits of sharing positive events. Journal of Personality and Social Psychology, 87, 228-245.

Harber, K. D., \& Cohen, D. J. (2005). The emotional broadcaster theory of social sharing. Journal of Language and Social Psychology, 24, 382-400.

Herbette, G., \& Rimé, B. (2004). Verbalization of emotion in chronic pain patients and their psychological adjustments. Journal of Health Psychology, 9, 661-676.

Laurenceau, J.-P., Barrett, L. F., \& Pietromonaco, P. R. (1998). Intimacy as an interpersonal process: The importance of self-disclosure, partner disclosure, and perceived partner responsiveness in interpersonal exchanges. Journal of Personality and Social Psychology, 74, 1238-1251.

Langston, C. A. (1994). Capitalizing on and coping with daily-life events: Expressive responses to positive events. Journal of Personality and Social Psychology, 67, 1112-1125.

Lewis, M. (2000). The emergence of human emotions. In M. Lewis \& J. Haviland (Eds.), Handbook of emotions (pp. 265-280). New York: Guilford Press.

Luminet, O., Bouts, P., Delie, F., Manstead, A. S. R., \& Rimé, B. (2000). Social sharing of emotion following exposure to a negatively valenced situation. Cognition and Emotion, 14, 661688.

Marques, J., Paez, D. \& Serra, A. (1997). Social sharing, emotional climate and the transgenerational transmission of memories: The Portuguese Colonial War. In J. Pennebaker, D. Paez, \& B. Rimé (Eds.), Collective memory of political events (pp. 253-276). Mahwah, NJ: Erlbaum.

Paez, D., Rimé, B., Martinez, F., \& Basabe, N. (2006). Effects of the social sharing of emotions in a Spanish sample after the March 11th bombing in Madrid. Paper submitted for publication.

Reis, H. T., \& Patrick, B. C. (1996). Attachment and intimacy: Component processes. In E. T. Higgins \& A. W. Kruglanski (Eds.), Social psychology: Handbook of basic principles (pp. 523-563). New York: Guilford Press.

Rimé, B., (in press). Interpersonal emotion regulation. In J. J. Gross (Ed.), Handbook of emotion regulation. New York: Guilford Publications.

Rimé, B. (2005). Le partage social des émotions [The social sharing of emotions]. Paris: Presses Universitaires de France.

Rimé, B. \& Christophe, V. (1997). How individual emotional episodes feed collective memory. In J. W. Pennebaker, D. Paez, \& B. Rimé (Eds.). Collective memory of political events: Social and psychological perspectives (pp. 131-146). Hillsdale, NJ: Erlbaum.

Rimé, B., Finkenauer, C., Luminet, O., Zech, E., \& Philippot, P. (1998). Social sharing of emotion: New evidence and new questions. In W. Stroebe \& M. Hewstone (Eds.), European review of social psychology (Vol. 9, pp. 145-189). Chichester, UK: John Wiley \& Sons.

Rimé, B., Mesquita, B., Philippot, P., \& Boca, S. (1991). Beyond the emotional event: Six studies on the social sharing of emotion. Cognition and Emotion, 5, 435-465.

Rimé, B., Philippot, P., Boca, S., \& Mesquita, B. (1992). Long lasting cognitive and social consequences of emotion: Social sharing and rumination. In W. Stroebe \& M. Hewstone (Eds.), European review of social psychology (Vol. 3, pp. 225-258). Chichester, UK: John Wiley \& Sons.

Singh-Manoux, A., \& Finkenauer, C. (2001). Cultural Variations in social sharing of emotions: An intercultural perspective on a universal phenomenon. Journal of Cross-Cultural Psychology, $32,647-661$.

Zech, E., \& Rimé, B. (2005). Is talking about an emotional experience helpful? Effects on emotional recovery and perceived benefits. Clinical Psychology and Psychotherapy, 12, 270-287. 
BERNARD RIMÉ, PhD, is a professor at the Department of Psychology of the University of Louvain, Louvain-la-Neuve, Belgium. He is the author of Le partage social des émotions (The Social Sharing of Emotion, 2005) and a coeditor of Les emotions (1989), Fundamentals of Nonverbal Behavior (1991), and Collective memory of political events: Social psychological perspectives (1997). He was President of ISRE (International Society for Research on Emotion) from 1997 to 2000. 


\section{Queries}

Q1 Author: Please note that "de Rivera \& Paez, this issue" is not given in the reference list. Kindly provide details of the same.

Q2 Author: Please note that the year "2000" in "Singh-Manoux \& Finkenauer, 2000 " has been changed to 2001 , in accordance with that given in the reference list.

Q3 Author: Please note that "Pennebaker and Harber (1993)" is not given in the reference list. Kindly provide details of the same.

Q4 Author: Please note that "Paez et al. in this issue" is not given in the reference list. Kindly provide details of the same.

Q5 Author: Please note that "Paez et al., this issue" is not given in the reference list. Kindly provide details of the same.

Q6 Author: Please note that "Lewis, 2000" is not cited anywhere in the text. Kindly insert its citation at an appropriate place in the text.

Q7 Author: Please update "Paez et al., 2006" if possible.

Q8 Author: Please update "Rimé, in press" if possible.

Q9 Author: Please note that "Rimé, in press" and "Rimé, 2005" are not cited anywhere in the text. Kindly insert their citations at appropriate places in the text. 